\title{
Prevalence of dementia in intellectual disability using different diagnostic criteria
}

\author{
A. STRYDOM, G. LIVINGSTON, M. KING and A. HASSIOTIS
}

\author{
Background Diagnosis of dementia is \\ complex in adults with intellectual \\ disability owing to their pre-existing \\ deficits and different presentation.
}

\begin{abstract}
Aims To describe the clinical features and prevalence of dementia and its subtypes, and to compare the concurrent validity of dementia criteria in older adults with intellectual disability.
\end{abstract}

Method The Becoming Older with Learning Disability (BOLD) memory study is a two-stage epidemiological survey of adults with intellectual disability without Down syndrome aged 60 years and older, with comprehensive assessment of people who screen positive. Dementia was diagnosed according to ICD-I0, DSM-IV and DC-LD criteria.

Results The DSM-IV dementia criteria were more inclusive. Diagnosis using ICD-10 excluded people with even moderate dementia. Clinical subtypes of dementia can be recognised in adults with intellectual disability. Alzheimer's dementia was the most common, with a prevalence of $8.6 \%$ (95\% Cl 5.2-13.0), almost three times greater than expected.

Conclusions Dementia is common in older adults with intellectual disability, but prevalence differs according to the diagnostic criteria used. This has implications for clinical practice.

Declaration of interest None.
Adults with intellectual disability are increasingly surviving to old age and are therefore vulnerable to age-associated disorders such as dementia. It is well known that adults with Down syndrome have a genetic risk of Alzheimer's dementia. Previous small epidemiological studies have indicated that dementia may also be common in the population of people with intellectual disability who do not have Down syndrome (Patel et al, 1993; Cooper, 1997). Moreover, there are indications that dementia presentation may differ in those with and without this syndrome (Cooper \& Prasher, 1998). It is therefore important to consider the population with intellectual disability without Down syndrome separately from those who do have the syndrome. Using standard diagnostic criteria is complicated because of both the premorbid cognitive deficits and the heterogeneity of ability in this population. The ICD-10 criteria (World Health Organization, 1993) have been recommended because they put more emphasis on non-cognitive aspects of dementia such as emotional lability and apathy, which are believed often to be the presenting signs of dementia in adults with intellectual disability (Aylward et al, 1997). Subsequently, the ICD-10 dementia criteria have been modified for this population and included in the Royal College of Psychiatrists' diagnostic criteria for psychiatric disorders in adults with learning disability (DC-LD; Royal College of Psychiatrists, 2001). The relative validity of dementia criteria has not been examined in the population with intellectual disability, neither has there been any study describing the prevalence of subtypes of dementia in older people with such disability. In this study we aimed to apply different dementia criteria in an epidemiological sample of older adults with intellectual disability, to describe the prevalence of dementia subtypes, to compare the different criteria and to describe the clinical features of those with dementia.

\section{METHOD}

The Becoming Older with Learning Disability (BOLD) memory study is a two-stage epidemiological survey of dementia in the total population of adults with intellectual disability without Down syndrome aged 60 years and older living in five London Boroughs; this area had a total adult population aged 60 years and older of 177544 people in the UK 2001 census (http:// www.statistics.gov.uk/census2001/census2001.asp). The protocol received approval from the Thames Valley Multi-centre Research Ethics Committee and from the Research and Development offices of all participating National Health Service (NHS) organisations.

\section{Participants}

We identified potential participants from social services' electronic databases of past and present intellectual disability service users combined with lists of past or present users of local intellectual disability health teams. We also contacted all residential and day services providers for adults with intellectual disability to ensure that all known older adults with such disability had been identified. Participants included those resident in their own homes, family homes, residential homes of all types, nursing homes and hospitals. In two of the boroughs we also contacted all geriatricians, old age psychiatrists, mental health teams for older people, and all residential and nursing homes caring for people without intellectual disability: this resulted in the identification of only one additional participant with intellectual disability, and so this extension of the sampling frame was not implemented in the other three boroughs. Participants received accessible information written in simple language with pictures. A capacity assessment was undertaken to determine whether the person was able to provide consent; if this was the case, written informed consent was obtained. For those who did not have capacity to consent, assent was given by carers, provided the person did not show unwillingness to participate. Written informed consent was also gained from informants for their own participation. We sought historical information to cover at least the preceding 2 years for those who screened positive.

Intellectual disability was defined according to ICD-10 criteria for mental retardation (World Health Organization, 
1993): that is, a reduced level of intellectual functioning (an IQ below 70) which first manifested during the developmental period and results in diminished ability to adapt to the daily demands of the normal social environment. Those in whom the diagnosis was uncertain underwent an assessment and were excluded if they did not meet the ICD-10 criteria. Adults with Down syndrome were identified from records of chromosomal analysis or by their characteristic features, and excluded from the study.

\section{Screening stage}

All participants who were able and all informants completed a screen for symptoms of dementia or cognitive decline. Informants completed the Dementia Questionnaire for Persons with Mental Retardation (DMR; Evenhuis, 1996), an established screening tool for dementia with good psychometric properties in this population (Strydom \& Hassiotis, 2003). They also completed a brief activities of daily living schedule based on the Adaptive Behavior Scale (Nihira et al, 1992) and the Activities for Daily Living Schedule (Lawton \& Brody, 1969). We recorded collected information about level of functioning in early life and decline in activities of daily living over the past 2 years from informants. Participants with intellectual disability who had sufficient communication ability completed a three-item object memory task based on the Shoe Box Test (Burt \& Aylward, 2000; Silverman et al, 2004). Screening criteria were designed for maximum sensitivity so that no person with dementia would be missed. Screen positives fulfilled any of the following conditions: a score at or above the cognitive score thresholds for dementia provided by Evenhuis (1996) for severe, high-moderate or mild intellectual disability on the DMR; an unexplained decline in activities of daily living; or a delayed recall of fewer than two items in the memory task. Participants who screened negative on these criteria were presumed not to have dementia.

\section{Assessment of people who screened positive}

Participants who screened positive completed a full assessment to elicit symptoms of dementia as described below.

All screening tests and assessments were completed by a qualified intellectual disability psychiatrist (A.S.).

\section{Neuropsychological assessment}

Basic neuropsychological assessment consisted of the Test for Severe Impairment (Albert \& Cohen, 1992), additional memory items from the Severe Impairment Battery (Saxton \& Swihart, 1989), the Tower of London test (Shallice, 1982), the Supermarket Fluency task (Troyer, 2000), the British Picture Vocabulary Scale (Dunn \& Dunn, 1997) and the Luria three-stage command (Hodges, 1994). Informants also completed a questionnaire based on a modification of the Cambridge Mental Disorders Examination (CAMDEX) informant questionnaire to elicit a history of changes in memory, personality, general cognitive function and confusion (Ball et al, 2004).

\section{Physical examination}

A structured physical examination was conducted to record neurological symptoms and signs associated with dementia and to identify other physical disease such as thyroid disease, neurological conditions and cardiovascular disorders, based on memory clinic assessments (Hassiotis et al, 2003). This included a vision and hearing screen. Informants provided details of current health and medications, and medical records were reviewed to obtain information on previous health status and recent investigations. We recorded the results of neuroimaging undertaken in the preceding 2 years.

\section{Mental state examination}

Mental disorders and psychiatric symptoms were screened for with the mini Psychiatric Assessment Schedule for Adults with Developmental Disability (PAS-ADD), a tool for assessing adults with intellectual disability (Moss, 2002).

\section{Diagnosis}

All the above information was compiled in an anonymised summary, which was presented to two of three psychiatrists (A.H., G.L. or A.S.) for independent diagnostic review. Two were intellectual disability psychiatrists and one (G.L.) was an old age psychiatrist. Any disagreement in ratings was settled by discussion with the third psychiatrist. A specially developed tick list with operationalised criteria was used to produce a differential diagnosis. We applied the following diagnostic principles:

(a) The key to dementia diagnosis in this population is decline in cognitive function from an individual baseline, not change from a normal level (Aylward et al, 1997).

(b) We followed a hierarchical process, consistent with diagnostic systems such as DC-LD (Royal College of Psychiatrists, 2001), whereby developmental level, mental retardation syndrome, autistic disorders, physical illness and medication effects, sensory loss, environmental change or life events, or mental illness had to be considered sequentially as possible reasons for screening positive.

(c) General dementia criteria had to be met first before moving on to subtyping. However, since criteria for Lewy body dementia and frontotemporal dementia were designed as stand-alone criteria outside of the ICD-10 or DSM-IV criteria, these disorders were not subjected to the two-stage process.

(d) The list included the following criteria for dementia: ICD-10 Research Diagnostic Criteria (World Health Organization, 1993), DSM-IV-TR (American Psychiatric Association, 2000) and DC-LD criteria (Royal College of Psychiatrists, 2001), which are compared in Table 1; ICD-10 (World Health Organization, 1993), DSM-IV (American Psychiatric Association, 2000) and the National Institute of Neurological and Communicative Disorders and Stroke-Alzheimer's Disease and Related Disorders Association (NINCDS-ADRDA; McKhann et al, 1984) criteria for Alzheimer's disease; ICD-10, DSM-IV and National Institute of Neurological Disorders and Stroke-Association Internationale pour la Recherche et l'Enseignement en Neurosciences NINDS-AIREN (Roman et al, 1993) criteria for vascular dementia; the Consortium on Dementia with Lewy Bodies (DLB) criteria (McKeith et al, 1996); and the Work Group on Frontotemporal Dementia and Pick's Disease criteria for frontotemporal dementia (FTD; McKhann et al, 2001).

(e) Dementia is an organic disorder and should therefore trump mental illnesses such as depression in hierarchical systems; instead, it is often defined as a diagnosis of exclusion in the diagnostic systems. We made the diagnosis of dementia in the presence of depressive symptoms if these were deemed not to account for the cognitive decline, but the final judgement 
Table I Criteria for dementia in the classification systems

\begin{tabular}{|c|c|c|c|}
\hline Impaired domain/ symptoms & DSM-IV & ICD-10 & DC-LD \\
\hline \multicolumn{4}{|l|}{ Memory } \\
\hline Short- and/or long-term & + & + & + \\
\hline \multicolumn{4}{|l|}{ Higher cortical functions ${ }^{2}$} \\
\hline Executive function & O & & \\
\hline Thinking & & O & O \\
\hline Judgement & & $\mathrm{O}$ & O \\
\hline Other cognitive skills & & & O \\
\hline Information processing & & $\mathrm{O}$ & \\
\hline Aphasia/ language skills & O & & \\
\hline Apraxia & O & & \\
\hline Agnosia & O & & \\
\hline \multicolumn{4}{|l|}{ Behavioural and emotional function ${ }^{2}$} \\
\hline Emotional lability & & O & O \\
\hline Irritability & & O & 0 \\
\hline Apathy & & $\mathrm{O}$ & O \\
\hline Social behaviour & & $\mathrm{O}$ & $\mathrm{O}$ \\
\hline \multicolumn{4}{|l|}{ Other criteria } \\
\hline Change from premorbid state/decline in level of functioning ${ }^{\prime}$ & + & & + \\
\hline Duration of at least 6 months & & + & + \\
\hline \multicolumn{4}{|l|}{ Exclusions } \\
\hline Not caused by delirium & + & + & + \\
\hline Not caused by mental illness & + & & + \\
\hline
\end{tabular}

+ , required for diagnosis.

I. The ICD-10 classification requires a decline in memory and other cognitive function, but does not have a separate criterion for change or deterioration in function.

2. At least one of the circled is required.

depended on how the diagnostic criteria were worded; e.g. ICD-10 does not have a mental illness exclusion criterion at all, whereas DC-LD has an exclusion for mental illness (dementia can only be diagnosed in the presence of mental illness if it is deemed not to account for the cognitive decline).

(f) It was possible to meet the criteria for more than one subtype of dementia unless it was an explicit exclusion. In practice, this meant that the vascular events associated with vascular dementia were exclusions for ICD-10 Alzheimer's disease and frontotemporal dementia. The NINCDS-ADRDA criteria allowed possible Alzheimer's disease in the presence of vascular disease, and DSM-IV allowed a diagnosis of Alzheimer's disease if the vascular events were judged not to be directly associated with the dementia.

(g) We included a clinical rating of dementia severity (mild, moderate or severe dementia).
For the purpose of this analysis the participants were divided into two groups: those with dementia (if they met any of the above diagnostic criteria) and those who did not meet the criteria.

\section{Statistical analysis}

Data were entered into the Statistical Package for the Social Sciences version 11 for Windows. Prevalence rates are presented in percentages, rounded to one decimal place. Symmetrical exact binomial 95\% confidence intervals were derived using a calculator available at http://statpages.org/ confint.html. Chi-squared tests were used to analyse categorical variables with continuity correction for $2 \times 2$ tables; Fisher's exact tests were used if $50 \%$ or more cells had expected values of less than 5. Significance level was set at $P<0.01$ owing to the number of tests; $t$-tests were used to analyse differences in mean age. Correlation between sets of criteria was calculated with Spearman's rho.

Prevalence rates for Alzheimer's disease and vascular dementia in the general population were obtained from the most recent European collaborative study of population-based cohorts $(4.4 \%$ for Alzheimer's disease and $1.6 \%$ for vascular dementia; Lobo et al, 2000). These rates were used to calculate expected counts for this study. The observed count divided by the expected count provided standardised morbidity ratios (SMRs) for comparison of rates between populations (Page et al, 1995). Confidence intervals for SMRs were obtained with a calculator providing exact $95 \%$ Poisson confidence intervals (http://home. clara.net/sisa/smr.htm).

\section{RESULTS}

We identified 258 potential participants from health or social services. An additional $23(8.2 \%)$ were identified through other providers. All 281 potential participants were contacted. Of these, $24(8.5 \%)$ were ineligible for the study because of unrecorded Down syndrome status, being too young, having died recently, not having an intellectual disability, or not residing at the given address. Of the remaining 257 individuals, $35(13.6 \%)$ refused participation, or their carers refused on their behalf; $222(86.4 \%)$ participated. The prevalence of eligible participants in the total population of all adults aged 60 years and older living in these boroughs was $0.15 \%$. Participants did not differ from non-participants in terms of mean age (68.8 v. 67.9 years; $t=0.776, \quad P=0.439$ ) or gender (Pearson $\left.\chi^{2}=0.14, P=0.708\right)$. The proportion of male to female participants was $52.7 \%$ to $47.3 \%$. With regard to severity of disability, $123(55.4 \%)$ participants were rated to have mild intellectual disability and $99(44.6 \%)$ had moderate or more severe disability.

\section{Participants who screened positive}

Overall, 60 people screened positive; 29 of these met at least one set of dementia criteria (including DLB and FTD criteria). Of these, $13(45 \%$, or $5.9 \%$ of the total $)$ already had the diagnosis of probable or possible dementia recorded in their clinical notes. 'False' positives (i.e. those who screened positive but did not meet dementia criteria) were younger (mean age 70.9 v. 76.4 years; $t=-2.667, P=0.01$ ) and more likely to have severe intellectual disability (41.9 v. 3.4\%; $\chi^{2}=10.349$, $P=0.001$ ), but the true positives and false positives did not differ significantly with 
regard to gender, health problems, mental illness or sensory disabilities.

\section{Mental illness}

The prevalence rates of current mental illness (as reported by informants or extracted from medical records) are given in Table 2; this table also includes the numbers with scores above the mini PAS-ADD thresholds. The proportions of those with mental illness who were also diagnosed with dementia are given in the last column. Since depression is an important differential diagnosis of dementia and may be difficult to distinguish from dementia in older adults, we examined all the cases with a history or mini PAS-ADD threshold score of depression that also met the criteria for dementia. Six adults with a recent history of depression were deemed to have dementia. Only two of them had scores above the depression threshold of the mini PASADD; the rest had fully recovered or had remission of most symptoms, and their cognitive declines were deemed not to relate to the depressive episode. Three of them met all three sets of dementia criteria; one met only the DSM-IV criteria because she did not have a history of behavioural or social decline. She was diagnosed with dementia due to Parkinson's disease. Of the adults who reached the mini PAS-ADD threshold for depression, one was a 69-year-old woman with mild intellectual disability and a long history of cognitive decline, considerable loss of function and emergence of other neuropsychiatric symptoms. She was diagnosed by her local intellectual disability psychiatrist as having Alzheimer's disease 2 years prior to participating in the study, and was treated with donepezil for 6 months. She was rated to have depression symptoms secondary to dementia and met the dementia criteria of ICD-10, DC-LD and DSM-IV. The other person was a 75year-old man with mild intellectual disability and a history of psychotic illness with depressive episodes since early adulthood. He had a 2-year history of gradual decline in cognitive function and activities of daily living, personality and behavioural changes, episodes of confusion and falls. He had memory deficits on psychometric testing and met the ICD-10 criteria for dementia, but not those of DSM-IV or DC-LD because the raters were not unable to exclude the possibility that his symptoms were related to his mental illness.

Table 2 Mental illness and dementia diagnoses

\begin{tabular}{llcc}
\hline & $n$ & $\begin{array}{c}\text { Prevalence } \\
(\%)\end{array}$ & $\begin{array}{c}\text { With } \\
\text { dementia } \\
n(\%))^{\prime}\end{array}$ \\
\hline Recent history of mental illness (n=222) & & & \\
$\quad$ Depression & 24 & 10.8 & $6(25)$ \\
Bipolar disorder & 10 & 4.5 & $2(20)$ \\
Anxiety disorder & 16 & 7.2 & $4(25)$ \\
Schizophrenia & 2 & 0.9 & $0(0)$ \\
Psychosis NOS & 15 & 6.8 & $7(47)$ \\
$\quad$ Any mental disorder (including behavioural problems) & 93 & 41.9 & $18(19)$ \\
Mini PAS-ADD cases (n=60) & & & \\
Depression & 2 & & $2(100)$ \\
Mania & 2 & & $1(50)$ \\
Anxiety & 9 & & $6(67)$ \\
Psychosis & 7 & & $5(71)$ \\
Unspecified disorder & 7 & & $7(100)$ \\
\hline
\end{tabular}

PAS-ADD, Psychiatric Assessment Schedule for Adults with Developmental Disabilities; NOS, not otherwise specified.

I. Percentage of group with that disorder.

2. Completed for those who screened positive only; prevalence rates therefore not available.

\section{Dementia symptoms}

There were 26 participants with dementia for whom the informants could identify the initial symptoms. The most common initial symptom was general deterioration in functioning $(n=13 ; 50 \%$ of those with dementia), followed by behavioural or emotional change $(n=4 ; 15.4 \%)$. Deterioration in memory $(n=2,7.7 \%)$ or other cognitive functions $(n=2 ; 7.7 \%)$ was rarely noticed to be prominent in the early stages of the disorder. Other early symptoms $(n=5)$ included episodes of confusion $(n=3)$.

We compared the current dementia symptoms reported by informants in those who screened positive by diagnostic group (any dementia compared with no dementia) (Table 3). The most common reported symptoms for those with dementia were decline in self-care $190 \%$ of those with dementia), decline in instrumental activities of daily living (72\%), memory decline $(73 \%)$, episodes of confusion $(52 \%)$ and the development of muddled thinking (62\%). Symptoms that significantly discriminated between those with and without dementia in those who screened positive were deterioration in self-care ability, deterioration in instrumental activities of daily living, change in memory, development of muddled thinking, development of problems with thinking ahead and planning, and newly developed perseveration. None of the behavioural and emotional symptoms was discriminative of dementia.

\section{Overall dementia and subtype prevalence rates}

Prevalence rates for dementia and subtype criteria are given in Table 4. Criteria for Alzheimer's disease (ICD-10, DSM-IV or NINCDS-ADRDA) were met in $66 \%$ of those with dementia. The second most common subtype was Lewy body dementia (possible and probable cases) followed by frontotemporal dementia and then vascular dementia. Frontotemporal dementia was the most common subtype after Alzheimer's disease if possible cases of Lewy body dementia are discounted. Alzheimer's and vascular dementias diagnosed by DSM-IV criteria were almost twice as common as the corresponding ICD-10 rates (Table 4). The prevalence rates for those aged 65 years or over who met any criteria for Alzheimer's or vascular dementia were used to make comparisons with the general population rates. The 17 observed cases of Alzheimer's disease among those aged 65 years or over compared with 6.25 expected cases resulted in a standardised morbidity ratio (SMR) of 2.72 (95\% CI 1.58-4.35). The corresponding observed $v$. expected count for vascular dementia was 5 v. 2.27 $(\mathrm{SMR}=2.20,95 \%$ CI $0.72-5.14)$.

\section{Dementia criteria}

Twenty-eight people met any of the ICD10, DSM-IV or DC-LD criteria for dementia; 27 of these (12.2\% of the total sample) 
Table 3 Dementia symptoms reported by informants (screen-positive cases; $n=60$ )

\begin{tabular}{|c|c|c|c|c|c|c|c|}
\hline & \multirow{2}{*}{$\begin{array}{c}\text { No } \\
\text { dementia } \\
n=31 \\
n(\%)\end{array}$} & \multicolumn{2}{|c|}{ Dementia on any criteria } & & \multirow{2}{*}{$\begin{array}{c}\text { No } \\
\text { dementia } \\
n=31 \\
n(\%)\end{array}$} & \multicolumn{2}{|c|}{ Dementia on any criteria } \\
\hline & & $\begin{array}{l}n=29 \\
n(\%)\end{array}$ & $\begin{array}{l}\text { Within dementia } \\
\text { group } \%\end{array}$ & & & $\begin{array}{l}n=29 \\
n(\%)\end{array}$ & $\begin{array}{c}\text { Within dementia } \\
\text { group } \%\end{array}$ \\
\hline Memory & & & & Irritable or angry & & & \\
\hline Change in memory** & & & & No & $21(68)$ & $10(32)$ & 35 \\
\hline No & $28(80)$ & $7(20)$ & 27 & More & $6(30)$ & $14(70)$ & 48 \\
\hline Yes & $0(0)$ & $19(100)$ & 73 & Less & $3(38)$ & $5(62)$ & 17 \\
\hline Executive function & & & & Changeable in mood & & & \\
\hline Decision difficulty & & & & No & $28(58)$ & $20(42)$ & 69 \\
\hline No & $24(56)$ & $19(44)$ & 76 & Yes & $3(25)$ & $9(75)$ & 31 \\
\hline Yes & $0(0)$ & $6(100)$ & 24 & Less concern for others & & & \\
\hline \multicolumn{2}{|c|}{ Thinking ahead/planning problems** } & & & No & $24(5 I)$ & $23(49)$ & 79 \\
\hline No & $26(63)$ & $15(37)$ & 54 & Yes & $0(0)$ & $6(100)$ & 21 \\
\hline Yes & I (7) & $13(93)$ & 46 & Embarrassing behaviour & & & \\
\hline \multirow{2}{*}{\multicolumn{2}{|c|}{$\begin{array}{l}\text { Other cognitive functions } \\
\text { Keep mind on things/concentration }\end{array}$}} & & & No & $25(50)$ & $25(50)$ & 86 \\
\hline & & & & Yes & $4(50)$ & $4(50)$ & 14 \\
\hline No & $24(62)$ & $15(38)$ & 54 & Stubbornness & & & \\
\hline Yes & $5(28)$ & $13(72)$ & 46 & No & $22(56)$ & $17(44)$ & 59 \\
\hline Muddled thinking** & & & & More & $7(39)$ & II (6I) & 38 \\
\hline No & $22(69)$ & $10(31)$ & 36 & Less & $I(50)$ & $\mathrm{I}(50)$ & 3 \\
\hline Yes & $5(22)$ & $18(78)$ & 62 & Decline in functional abilities & & & \\
\hline Talking more or less & & & & Self-care ability decline** & & & \\
\hline No & $22(59)$ & $15(4 I)$ & 52 & No & $16(84)$ & $3(16)$ & 10 \\
\hline Yes & $9(39)$ & $14(61)$ & 48 & Yes & $15(37)$ & $26(63)$ & 90 \\
\hline Word-finding difficulty & & & & Instrumental ADL decline** & & & \\
\hline No & $28(6 I)$ & $18(39)$ & 67 & No & $24(75)$ & $8(25)$ & 28 \\
\hline Yes & $I(10)$ & $9(90)$ & 33 & Yes & $7(25)$ & $21(75)$ & 72 \\
\hline Perseveration** & & & & Social function change & & & \\
\hline No & $29(62)$ & $18(38)$ & 64 & No & $25(61)$ & $16(39)$ & 55 \\
\hline Yes & I (9) & $10(91)$ & 36 & Yes & $6(32)$ & $13(68)$ & 45 \\
\hline \multicolumn{2}{|c|}{ Behavioral and emotional functions } & & & Other symptoms & & & \\
\hline More impulsive & & & & Hallucinations & & & \\
\hline No & $27(5 \mathrm{l})$ & $26(49)$ & 93 & No & $28(55)$ & $23(45)$ & 79 \\
\hline Yes & $2(50)$ & $2(50)$ & 7 & Yes & $3(33)$ & $6(67)$ & 21 \\
\hline Character change & & & & Episodes of confusion & & & \\
\hline No & 31 (53) & $27(47)$ & 93 & No & $25(64)$ & $14(35)$ & 48 \\
\hline Yes & $0(0)$ & $2(100)$ & 7 & Yes & $6(29)$ & $15(7 \mid)$ & 52 \\
\hline
\end{tabular}

$A D L$, activities of daily living.

$* * P<0.01 ; \chi^{2}$ tests with continuity correction and I degree of freedom

met the criteria for DSM-IV dementia, 22 $(9.9 \%)$ met the criteria for ICD-10 dementia and $23(10.4 \%)$ the criteria for DC-LD dementia. The overlap between these criteria is shown in Fig. 1: this demonstrates that 21 participants $(75 \%)$ met all three sets of criteria, those meeting DC-LD criteria were a subset of those meeting DSM-IV criteria, and there were 5 participants who met one set of diagnostic criteria only (ICD-10 or DSM-IV). The criteria are therefore correlated as follows: DSM-IV $\times$ ICD-10 $\quad r=0.772 \quad(P \leqslant 0.005)$; DSM-IV $\times$ DC-LD $\quad r=0.872 \quad(P \leqslant 0.005)$; DC-LD $\times$ ICD-10 $r=0.894(P \leqslant 0.005)$.

The raters made clinical ratings of severity of dementia for all 29 meeting at least one set of criteria: $12(41 \%)$ were rated as having mild dementia, $16(55 \%)$ as having moderate dementia and $1(3 \%)$ as having severe dementia. Those diagnosed according to ICD-10 and DSM-IV dementia criteria were compared according to severity of dementia. Ten $(83 \%)$ of the 12 rated as having mild dementia met DSM-IV criteria compared with 8 $(66.7 \%)$ who met ICD-10 criteria. Six people met criteria for DSM-IV dementia but not ICD-10, and one met the criteria for ICD-10 but not DSM-IV. Of the six diagnosed by DSM-IV but not by ICD10 , half were rated clinically to have dementia of moderate severity. These were 
Table 4 Prevalence rates for dementia subtypes

\begin{tabular}{|c|c|c|c|c|c|c|}
\hline \multirow[t]{2}{*}{ Dementia subtype } & \multicolumn{3}{|c|}{$\begin{array}{c}\text { Age } \geqslant 60 \text { years } \\
(n=222)\end{array}$} & \multicolumn{3}{|c|}{$\begin{array}{c}\text { Age } \geqslant 65 \text { years } \\
(n=142)\end{array}$} \\
\hline & $n$ & $\%$ & $95 \% \mathrm{Cl}$ & $n$ & $\%$ & $95 \% \mathrm{Cl}$ \\
\hline \multicolumn{7}{|l|}{ Alzheimer's dementia } \\
\hline Any criteria & 19 & 8.6 & $5.2-13.0$ & 17 & 12 & $7.1-18.5$ \\
\hline \multicolumn{7}{|l|}{ Specific criteria } \\
\hline ICD-10 & 8 & 3.6 & $1.6-7.0$ & 8 & 5.6 & $2.5-10.8$ \\
\hline DSM-IV & 14 & 6.3 & $3.5-10.4$ & 12 & 8.5 & $4.4-14.3$ \\
\hline NINCDS-ADRDA & 12 & 5.5 & $2.8-9.3$ & II & 7.7 & $3.9-13.4$ \\
\hline \multicolumn{7}{|l|}{ Vascular dementia } \\
\hline Any criteria & 6 & 2.7 & $1.0-5.8$ & 5 & 3.5 & $1.2-8.0$ \\
\hline \multicolumn{7}{|l|}{ Specific criteria } \\
\hline ICD-10 & 3 & 1.4 & $0.3-3.9$ & 2 & 1.4 & $0.2-5.0$ \\
\hline DSM-IV & 6 & 2.7 & $1.0-5.8$ & 5 & 3.5 & $1.2-8.0$ \\
\hline NINDS-AIREN & 6 & 2.7 & $1.0-5.8$ & 5 & 3.5 & $1.2-8.0$ \\
\hline \multicolumn{7}{|l|}{ Dementia of Lewy body type } \\
\hline Any criteria & 13 & 5.9 & $3.2-9.8$ & II & 7.7 & $3.9-13.4$ \\
\hline \multicolumn{7}{|l|}{ DLB Consortium criteria } \\
\hline Possible & 9 & 4.1 & $1.9-7.6$ & 8 & 5.6 & $2.5-10.8$ \\
\hline Probable & 4 & 1.8 & $0.5-4.6$ & 3 & 2.1 & $0.4-6.0$ \\
\hline \multicolumn{7}{|l|}{ Frontotemporal dementia } \\
\hline FTD Work Group criteria & 7 & 3.2 & $1.3-6.4$ & 6 & 4.2 & $1.6-9.0$ \\
\hline \multicolumn{7}{|l|}{$\begin{array}{l}\text { Other dementias (e.g. head } \\
\text { trauma and Parkinson's disease) }\end{array}$} \\
\hline Any criteria & 3 & 1.4 & $0.3-3.9$ & 2 & 1.4 & $0.4-6.0$ \\
\hline Any dementia & 29 & 13.1 & $8.9-18.2$ & 26 & 18.3 & 12.3-25.7 \\
\hline
\end{tabular}

DLB, dementia with Lewy bodies; FTD, frontotemporal dementia.

excluded from ICD-10 criteria either because informant history of memory decline was absent (as opposed to other evidence of such decline, which is acceptable for DSM-IV diagnosis) or by the absence of behavioural and emotional symptoms. The extra ICD-10 case was rated to have mild dementia. The reason this did not meet DSM-IV criteria was that depressive symptoms were present and therefore one of the DSM-IV exclusion criteria was met.

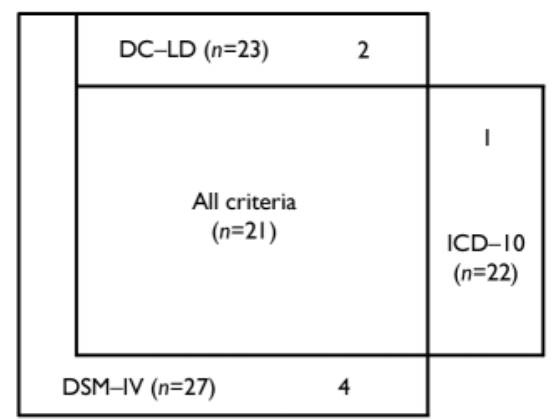

Fig. I Venn diagram of participants diagnosed with dementia on different diagnostic criteria. be due to the low prevalence of some (but not all) vascular risk factors such as smoking in the population with intellectual disability (Janicki et al, 2002).

Participants with dementia were reported by their carers to have had initial deterioration in functional ability rather than changes in memory and other cognitive functions. Non-cognitive symptoms such as personality changes were also common early symptoms, but did not differentiate between those with and without dementia who screened positive. Deterioration in self-care ability and instrumental activities of daily living were both discriminative of dementia in people who screened positive.

Because dementia may present differently in this population compared with the general population, criteria for the disorder may also perform differently. This is the first study to make a detailed comparison of dementia criteria in older adults with intellectual disability. We have demonstrated that correlations between the ICD-10, DSM-IV and DC-LD dementia criteria were good, but there were important differences. The DSM-IV criteria diagnosed a larger number of participants with mild dementia than ICD-10 criteria and were therefore more inclusive. The ICD-10 criteria excluded not only those with mild dementia, but also a considerable proportion of those with moderate-tosevere dementia.

\section{DISCUSSION}

This is the first study to report the prevalence of subtypes of dementia, including frontotemporal and Lewy body dementia, in older adults with intellectual disability. We have demonstrated that the symptoms associated with all dementia subtypes can be recognised in older adults with such disability. As in their general population counterparts, Alzheimer's disease was the most common diagnosis, but with a prevalence of almost three times higher than expected. Lewy body and frontotemporal dementias were common, as in the general population (Stevens et al, 2002). However, these dementias were more common than vascular dementia, which is unexpected since vascular dementia is usually the second most common type in the general population (Fratiglioni et al, 2000). This may be due to the criteria for frontotemporal dementia we have used, which are broad and expected to be more sensitive than other criteria (Neary et al, 2005), but may also

\section{Limitations}

This study is the largest cross-sectional survey of dementia in the intellectual disability population to date; our sample represents approximately $1 \%$ of the estimated 26000 adults aged 60 years and over known to have intellectual disability in England (Emerson \& Hatton, 2004). We employed epidemiological sampling methods and achieved high participation rates. We identified all older adults known to have intellectual disability. Participants underwent a very sensitive screening strategy, and were fully assessed with established assessment methods and tools if screened positive, before we applied a rigorous diagnostic procedure, which incorporated the main diagnostic criteria for dementia.

Despite the comprehensive recruitment strategy, it is possible that we have missed some older adults with intellectual disability who were unknown to social or health services. However, we believe this number 
to be small because older adults with such disability are likely to need assistance owing to the functional problems associated with ageing. This is more likely to be provided by agencies outside the family because informal support networks decrease as people grow older. Furthermore, the comprehensive care system in the UK promotes formal assistance. A small number of potential participants unknown to any service might reduce the increased prevalence of Alzheimer's disease when compared with the general population, but is unlikely to change our main findings about the relative prevalence of subtypes, presentation of dementia or performance of diagnostic criteria. We excluded adults with Down syndrome recognised by their clinical features, but did not undertake chromosomal analysis; it is therefore possible that some of these excluded adults did not have trisomy 21.

Another limitation is that crosssectional assessments are less reliable than sequential assessments. We therefore supplemented our data with historical information from informants and medical records. Nevertheless, for a proportion of participants we were unable to decide whether or not they had dementia owing to insufficient data; these were included in the group without dementia. Our study might therefore have underestimated the true prevalence of subtypes of dementia.

\section{Dementia symptoms and concurrent validity of dementia criteria}

Because of diagnostic difficulties in this population, clinical diagnosis cannot be used as the gold standard for comparison. We therefore determined the correlation of different dementia criteria and demonstrated their utility, but also highlighted particular issues. Cognitive deficits are difficult to demonstrate in adults with limited verbal and functional ability (Burt \& Aylward, 1999); clinicians therefore often rely on informant reports of change. Our data confirm that change in memory and higher functions are not noticed early in people with intellectual disability, and because these changes are required for dementia diagnosis, adults with both intellectual disability and dementia may be diagnosed later in the course of the disorder when these changes have become more apparent.
Dementia criteria differ considerably and therefore yield widely differing prevalence rates in the general population (Ballard \& Bannister, 2005). The ICD-10 criteria are more specific but less sensitive than DSM-III-R or DSM-IV criteria (Erkinjuntti et al, 1997). We have shown that this is also the case in older adults with intellectual disability. One of the reasons for this is that ICD-10 criteria are more demanding to apply because they are more dependent on reliable information from informants (Henderson et al, 1994). Another limitation of the ICD-10 and DC-LD criteria is that behavioural and emotional changes are an additional required symptom for ICD-10 and DC-LD dementia, but not for DSM-IV dementia. These were reported to have occurred early in a small but significant number of adults with intellectual disability and dementia. However, these symptoms were not good at discriminating between those with and without dementia, and limit the number of people diagnosed with ICD-10 criteria. Even those clinically rated to have moderate severity of dementia did not meet ICD-10 criteria. This was contrary to the expectation of an international consensus group (Aylward $e t$ al, 1997).

The 'false' screen positives need special mention. Those with severe intellectual disability were more likely to meet screening criteria but not diagnostic criteria for dementia. The proportion of false screen positives may seem high, but a recent study in an elderly population noted that of 96 people with confirmed cognitive and functional impairment, only 55 satisfied the DSM-IV criteria for dementia (Shaji et al, 2005). These authors felt that the DSM-IV prevalence of dementia is possibly an underestimation; this might also be the case in the population with intellectual disability, because the 'false' screen positive group might contain cases of dementia that did not meet criteria owing to lack of informant or medical history, or to the difficulty of making this diagnosis in a group with severe disability.

\section{Clinical implications}

We found that more than double the number of older adults with intellectual disability meet dementia criteria than is recognised by their carers or health professionals. Functional decline was reported to be more common than memory decline early on in the presentation; perhaps the potential for pathological causes underlying such decline is not recognised in adults with lifelong deficits. Dementia should always be considered as a possible diagnosis when investigating reports of decline in older adults with intellectual disability. Our findings also give credence to screening approaches that rely on functional change (Prasher et al, 2004).

We preferred the DSM-IV criteria for dementia in this population. They are clearly set out and easy to interpret. They do not rely exclusively on informant report of memory and cognitive change like the ICD-10 criteria, which allows the clinician to use other sources of information such as sequential cognitive assessments and medical records. Furthermore, they do not require behavioural or emotional change but focus on functional change, which is important in this population. This has important implications for patients, since the use of DSM-IV criteria may enable earlier diagnosis of dementia in larger numbers of older adults with intellectual disability, which could gain them timely access to appropriate interventions.

\section{Future research}

Our findings raise questions about the aetiology of dementia in older adults with intellectual disability but without Down syndrome. It is important to establish why Alzheimer's dementia may be more common in these adults than in the general population; we have estimated an SMR of 2.72 (95\% CI 1.58-4.35). Possibilities include genetic causes such as apolipoprotein E4 alleles, or environmental causes such as brain damage during birth and early life, which is associated with intellectual disability but may also in the long term be associated with Alzheimer's disease.

The incidence and presentation of dementia and validity of diagnoses should be confirmed longitudinally. It is also important to confirm subtype diagnoses with post-mortem studies, and to investigate the aetiology of dementia in this population. This will enable appropriate interventions and illuminate our understanding of dementia presentation and progression throughout the intellectual range.

\section{ACKNOWLEDGEMENTS}

This study was funded by the Medical Research Council (UK) with a Training Fellowship Grant to AS. Additional support was provided by the Penrose Society in the form of a Jancar Travelling Fellowship 
to A.S. The research was also supported by the Research and Development departments of Oxleas National Health Service (NHS) Trust, Harrow Primary Care Trust (PCT), Enfield PCT, Islington PCT and Camden PCT, who all receive a proportion of funding from the NHS Executive; the views expressed in this publication are those of the authors and not necessarily those of the NHS Executive. The authors wish to thank all the participants and their carers, and local area clinicians and social services staff who enabled this study. Our protocol benefited from discussions with Professor Tony Holland, and training to A.S. by Mr Paul Patti, Dr Arthur Dalton and others at the New York State Institute for Basic Research in Developmental Disabilities.

\section{REFERENCES}

Albert, M. \& Cohen, C. (1992) The Test for Severe Impairment: an instrument for the assessment of patients with severe cognitive dysfunction. Journal of the American Geriatrics Society, 40, 449-453.

American Psychiatric Association (2000) Diagnostic and Statistical Manual of Mental Disorders (4th edn, revised) (DSM-IV-TR). APA.

Aylward, E. H., Burt, D. B., Thorpe, L. U., et al (1997) Diagnosis of dementia in individuals with intellectual disability. Journal of Intellectual Disability Research, $\mathbf{4 I}$ 152-164.

Ball, S. L., Holland, A. J., Huppert, F. A., et al (2004) The modified CAMDEX informant interview is a valid and reliable tool for use in the diagnosis of dementia in adults with Down's syndrome. Journal of Intellectual Disability Research, 48, 611-620.

Ballard, C. \& Bannister, C. (2005) Criteria for the diagnosis of dementia. In Dementia (eds A. Burns, J. O'Brien \& D. Ames), pp. 24-37. Arnold

Burt, D. B. \& Aylward, E. H. (1999) Assessment methods for diagnosis of dementia. In Dementia, Aging, and Intellectual Disabilities: A Handbook (eds M. P. Janick \& A. J. Dalton), pp. |4|-156. Brunner/Mazel.

Burt, D. B. \& Aylward, E. H. (2000) Test battery for the diagnosis of dementia in individuals with intellectual disability. Journal of Intellectual Disability Research, 44, 175-180.

Cooper, S. A. (1997) High prevalence of dementia among people with learning disabilities not attributable to Down's syndrome. Psychological Medicine, 27, 609-616.

Cooper, S. A. \& Prasher, V. P. (1998) Maladaptive behaviours and symptoms of dementia in adults with Down's syndrome compared with adults with intellectual disability of other aetiologies. Journal of Intellectual Disability Research, 42, 293-300.

Dunn, L. M. \& Dunn, L. M. (1997) British Picture Vocabulary Scale (2nd edn). nferNelson.

Emerson, E. \& Hatton, C. (2004) Estimating the Current Need/ Demand for Supports for People with Learning Disabilities in England. Institute for Health Research, Lancaster University.

Erkinjuntti, T., Ostbye, T., Steenhuis, R., et al (1997) The effect of different diagnostic criteria on the

A. STRYDOM, MBChB, MRCPsych, MSc, G. LIVINGSTON, MBChB, FRCPsych, MD, M. KING, MBChB, FRCPsych, PhD, A. HASSIOTIS, MA, MRCPsych, PhD, Department of Mental Health Sciences, Royal Free and University College Medical School, London, UK

Correspondence: Dr A. Strydom, Department of Mental Health Sciences, Royal Free and University College Medical School, UCL Hampstead Campus, London NW3 2PF, UK. Tel: +44 (0)20 77940500 , ext. 34120; fax: +44(0)207830 2808; email: a.strydom@medsch.ucl.ac.uk

(First received 17 July 2006, final revision 18 February 2007, accepted 26 March 2007)

prevalence of dementia. New England Journal of Medicine, 337, 1667-1674.

Evenhuis, H. M. (1996) Further evaluation of the Dementia Questionnaire for Persons with Mental Retardation (DMR). Journal of Intellectual Disability Research, 40, 369-373.

Fratiglioni, L., Launer, L. J., Andersen, K., et al (2000) Incidence of dementia and major subtypes in Europe: a collaborative study of population-based cohorts. Neurology, 54, SIO-SI5.

Hassiotis, A., Strydom, A., Allen, K., et al (2003) A memory clinic for older people with intellectual disabilities. Aging and Mental Health, 7, 418-423.

Henderson, A. S., Jorm, A. F., Mackinnon, A., et al (1994) A survey of dementia in the Canberra population - experience with ICD-10 and DSM-III-R criteria. Psychological Medicine, 24, 473-482.

Hodges, J. R. (1994) Testing cognitive function at the bedside. In Cognitive Assessment for Clinicians. pp. 108-154. Oxford University Press.

Janicki, M. P., Davidson, P.W., Henderson, C. M., et a (2002) Health characteristics and health services utilization in older adults with intellectual disability living in community residences. Journal of Intellectual Disability Research, 46, 287-298.

Lawton, M. P. \& Brody, E. M. (1969) Assessment of older people: self-maintaining and instrumental activities of daily living. Gerontologist, 9, 179-186.

Lobo, A., Launer, L. J., Fratiglioni, L., et al (2000) Prevalence of dementia and major subtypes in Europe: a collaborative study of population-based cohorts. Neurology, 54, S4-S9.

McKeith, I. G., Galasko, D., Kosaka, K., et al (1996) Consensus guidelines for the clinical and pathologic diagnosis of dementia with Lewy bodies (DLB): report of the consortium on DLB international workshop. Neurology, 47, III3-II24.

McKhann, G., Drachman, D., Folstein, M., et al (1984) Clinical diagnosis of Alzheimer's disease: report of the NINCDS-ADRDA Work Group under the auspices of Department of Health and Human Services Task Force on Alzheimer's Disease. Neurology, 34, 939-944.

McKhann, G. M., Albert, M. S., Grossman, M. et al (200I) Clinical and pathological diagnosis of frontotemporal dementia: report of the Work Group on Frontotemporal Dementia and Pick's Disease. Archives of Neurology, 58, 1803-1809.

Moss, S. (2002) The Mini-PASADD Interview Pack. Pavilion.
Neary, D., Snowden, J. \& Mann, D. (2005)

Frontotemporal dementia. Lancet Neurology, 4, 77I-780.

Nihira, K., Leland, L. \& Lambert, N. (1992) Adaptive Behavior Scale - Residential and Community (2nd edn). Pro-ed.

Page, R. M., Cole, G. E. \& Timmreck, T. C. (1995) Basic Epidemiological Methods and Biostatistics: A Practical Guidebook. Jones \& Bartlett.

Patel, P., Goldberg, D. \& Moss, S. (1993) Psychiatric morbidity in older people with moderate and severe learning disability. II: The prevalence study. British Journal of Psychiatry, 163, 48I-49|.

Prasher, V., Farooq, A. \& Holder, R. (2004) The Adaptive Behaviour Dementia Questionnaire (ABDQ) screening questionnaire for dementia in Alzheimer's disease in adults with Down syndrome. Research in Developmental Disabilities, 25, 385-397.

Roman, G. C., Tatemichi, T. K., Erkinjuntti, T., et al (1993) Vascular dementia: diagnostic criteria for research studies. Report of the NINDS-AIREN International Workshop. Neurology, 43, 250-260.

Royal College of Psychiatrists (200I) DC-LD: Diagnostic criteria for Psychiatric Disorders for Use with Adults with Learning Disabilities/Mental Retardation. (Occasional Paper OP48). Royal College of Psychiatrists.

\section{Saxton, J. \& Swihart, A. A. (1989)}

Neuropsychological assessment of the severely impaired elderly patient. Clinics in Geriatric Medicine, 5, 531-543.

Shaji, S., Bose, S. \& Verghese, A. (2005) Prevalence of dementia in an urban population in Kerala, India. British Journal of Psychiatry, 186, I36-140.

Shallice, T. (1982) Specific impairments of planning. Philosophical Transactions of the Royal Society of London. Series B Biological Sciences, 298, 199-209.

Silverman, W., Schupf, N., Zigman, W., et al (2004) Dementia in adults with mental retardation: assessment at a single point in time. American Journal on Mental Retardation, 109, III-125.

Stevens, T., Livingston, G., Kitchen, G., et al (2002) Islington study of dementia subtypes in the community British Journal of Psychiatry, 180, 270-276.

Strydom, A. \& Hassiotis, A. (2003) Diagnostic instruments for dementia in older people with intellectual disability in clinical practice. Aging and Mental Health, 7, 431-437.

Troyer, A. K. (2000) Normative data for clustering and switching on verbal fluency tasks. Journal of Clinical and Experimental Neuropsychology, 22, 370-378.

World Health Organization (1993) The ICD-10 Classification of Mental and Behavioural Disorders: Diagnostic Criteria for Research.WHO. 